\title{
The evolution of morbidity and mortality conferences
}

\author{
Darlene Tad-y, Heidi L Wald
}

University of Colorado, School of Medicine, Aurora, Colorado, USA

Correspondence to Dr Darlene Tad-y, University of Colorado, School of Medicine, Aurora, CO 80045, USA; darlene.tad-y@ucdenver.edu

Accepted 1 September 2016 Published Online First 21 September 2016

\section{SLinked}

http://dx.doi.org/10.1136/ bmjqs-2016-005459

\section{CrossMark}

To cite: Tad-y D, Wald HL. BMJ Qual Saf 2017:26:433-435.
Morbidity and mortality conferences (MMCs) have become a vital element of patient care, sitting at the intersection of medical education, quality improvement and risk management. MMCs may have increased in importance as a staple of safety education since the Accreditation Council for Graduate Medical Education has identified that the discussion and analysis of adverse events in a structured fashion promotes the learning of key quality and safety concepts. ${ }^{1-3}$ Groups across specialties and disciplines have implemented innovative models of MMCs as a vehicle to engage clinicians in discussions to learn from adverse events and to identify opportunities to improve care. In studying these new models, it has become clear that deliberate attention to the structure, processes and content of the conference yields the greatest opportunity for improving the quality of patient care beyond just learning the concepts of quality and safety. ${ }^{4} 5$ We now face the next iteration of the MMC and are tasked with describing the facets that will best allow MMCs to drive learning and improved outcomes.

In this issue, Kwok and colleagues highlight the impact of implementing a structured MMC, the Ottawa M\&M Model ('OM3 model'), at their acute care tertiary centre across multiple specialties. $^{6}$ The model consists of five key elements, including appropriate case selection, structured case analysis, the creation of and dissemination of bottomline summaries, the development of effective pathways for action items and interprofessional and multidisciplinary participation. The authors conducted a yearlong study of 16 clinical groups implementing the OM3 model. The investigators provided an OM3 toolkit that included relevant educational materials, dedicated coaching to the teams, encouraged the groups to establish a quality committee for subsequent action items and identified a specific champion for the MMC.

The authors primarily sought to assess the improvement in the quality of MMCs as measured by the overall OM3 index, a scoring system they created based on the key elements of the OM3 model. ${ }^{6}$ They applied the index to the MMCs of the participating groups prior to and after the intervention. Their secondary outcomes included awareness of the principles by attendees, changes in clinical policy and procedures as a direct result of the MMCs, perception of effectiveness of MMCs on quality of care, perception of the impact of the OM3 structure on the group environment, culture, existing processes and finally identification of success factors and barriers to implementation.

Their study demonstrated a significant improvement in the OM3 scoring index for participating teams. The median index increased from $12 / 24$ to $20 / 24$ for teams, with a greater degree of improvement for the surgical versus non-surgical teams. All elements of the score improved, except for the frequency of MMCs. Both online and in-person survey results showed participants generally felt that the elements of the OM3 restructure were implemented and were well received. Importantly, attendees felt that having the structure provided the opportunity to have improved discussions. Barriers identified through the surveys included lack of time, like a formal training in patient safety, and persistent cultural resistance to the change.

The work presented by these authors highlights both the progress and the opportunities in the evolution of the MMC. While demonstrating that a structured model for MMCs can be successfully deployed across specialties, the work also exposes some of the areas that still need to be developed.

First, it is difficult to measure the quality of MMCs. The authors recruited 
relevant experts to devise the OM3 index, based on the discrete elements of the structure that they propose. While a scoring system is useful in considering the success of MMCs, it begs the question: what are the measures of success for MMCs? Some have suggested that having a formalised structure and the subsequent discussion and analysis of adverse events as processes can improve patient safety. ${ }^{4} 7$

One group examined 42 MMCs at their institution and using an effectiveness index based on the number and completion of improvement initiatives deriving from MMCs. They identified that more effective MMCs are associated with the presentation of cases in a standardised fashion, using visual aids and literature and the thorough analysis of adverse events. Importantly, this also included the monitoring of previously decided actions. ${ }^{8}$ It is then logical to conclude that a structured MMC, including in-depth analysis of adverse events would lead to a greater number of action items, ultimately leading to the completion of novel improvement initiatives derived specifically from that MMC.

Taking this one step further, it would be important to study the degree to which these initiatives lead to policy changes and ultimately to improved patient outcomes. Even when MMCs succeed in identifying important system problems or latent errors in need of attention, there remains the far more difficult task of solving these problems. As with any safety monitoring strategy, identifying safety problems is the relatively easy part, the real work begins with following up on these cases with meaningful improvements. Long-term attention to the final impact on patients' outcomes related to initiatives arising from well-executed analyses in MMCs is required and would be the best measure of an MMC's success.

Second, the authors of this paper and many other published works describing MMCs continue to highlight the critical role of culture as both a promoter and a barrier to a successful MMC contributing to patient safety. Indeed, a culture of safety has been thought to be the bedrock for high-reliability organisations, associated with safety-promoting behaviours such as error reporting, reductions in adverse events and reduced mortality. ${ }^{10}$ Two recent studies examined this question more precisely. One found no association between the patient safety culture as measured by the Hospital Survey on Patient Safety Culture and successful collaboratives focused on improving catheterassociated infection rates and central-line-associated bloodstream infections rates. ${ }^{11}$ And, a systematic review of the literature conducted showed a statistically significant relationship between patient safety culture and adherence to standard precautions. ${ }^{12}$ It is worth noting that the instruments used in all of these studies to measure patient safety culture differed across the studies.

Again, we face the challenge of objectively measuring something as complex as patient safety culture.
Furthermore, we need a better understanding of the relationship between MMCs and patient safety culture -is it the lack of a patient safety culture that prevents MMCs from success? At the Department of Medicine, University of Colorado, we have used the development and dissemination of a revamped MMC model as a key component in our strategy to become a learning organisation with a strong safety culture. After a 2.5-year MMC initiative, faculty disagreement with the statement 'staff feel like their mistakes are held against them' increased from $13 \%$ to $60 \%$. And, faculty agreement with the question 'we are given feedback about changes put into place based on event reports' increased from $7 \%$ to $29 \% .{ }^{13}$ These preliminary results raise an interesting possibility that a successful MMC process could drive improvements in patient safety culture.

Finally, beyond just the discussion and analysis of adverse events, MMCs should help guide the determination of accountability for medical error. Despite more than a decade of work in patient safety, there remains a good deal of work to be done. ${ }^{14}$ While MMCs traditionally focused on assigning blame to individuals, discussions about 'collective accountability' have emerged, consistent with the concept that systems factors play a large role in the provision of unsafe care. ${ }^{15}$ The shift towards focusing on systems factors has led MMCs away from considering more deeply the role of individual clinicians. The accountability for the group versus individuals for medical errors needs to be balanced well, ${ }^{16}$ and MMCs should therefore provide ample investigation of the thought process of individual clinicians as well as the systems factors. In addition to identifying the types of cognitive errors as part of a structured analysis, MMCs could also incorporate instruction on metacognition, awareness of heuristics and discussing strategies for mitigating cognitive errors. ${ }^{17-19}$

MMCs have evolved beyond just an educational conference to an important tool in the broader strategy for improving patient safety and quality improvement. Looking ahead, the utility of the MMC lies in disseminating what works well and by better defining successful MMCs, understanding the relationship between MMCs and patient safety culture and incorporating more investigation of cognitive processes.

Competing interests None declared.

Provenance and peer review Commissioned; internally peer reviewed.

\section{REFERENCES}

1 Gonzalo JD, Yang JJ, Huang GC. Systems-based content in medical morbidity and mortality conferences: a decade of change. J Grad Med Educ 2012;4:438-44.

2 Bechtold ML, Scott S, Nelson K, et al. Educational quality improvement report: outcomes from a revised morbidity and mortality format that emphasised patient safety. Qual Saf Health Care 2007;16:422-7. 
3 Education ACFGM. ACGME Common Program Requirements. acgme.org. http://www.acgme.org/acgmeweb/Portals/0/PFAssets/ ProgramRequirements/CPRs2013.pdf (accessed 17 Dec 2014).

4 Bal G, Sellier E, Tchouda SD, et al. Improving quality of care and patient safety through morbidity and mortality conferences. J Healthc Qual 2014;36:29-36.

5 Deis JN, Smith KM, Warren MD, et al. Transforming the Morbidity and Mortality Conference into an Instrument for Systemwide Improvement. Advances in Patient Safety: New Directions and Alternative Approaches. 2008;(Vol. 2: Culture and Redesign).

6 Kwok ES, Calder LA, Barlow-Krelina E, et al. Implementation of a structured hospital-wide morbidity and mortality rounds model. BMJ Qual Saf 2017;26:439-48.

7 Lecoanet A, Vidal-Trecan G, Prate F, et al. Assessment of the contribution of morbidity and mortality conferences to quality and safety improvement: a survey of participants' perceptions. BMC Health Serv Res 2016;16:176.

8 François P, Prate F, Vidal-Trecan G, et al. Characteristics of morbidity and mortality conferences associated with the implementation of patient safety improvement initiatives, an observational study. BMC Health Serv Res 2016;16:35.

9 CNE KHPCMR. Culture of Safety. Nursing Clinics of NA 2015;50:139-52.

10 Weaver SJ, Lubomksi LH, Wilson RF, et al. Promoting a culture of safety as a patient safety strategy: a systematic review. Ann Intern Med 2013;158Pt 2):369-74.

11 Meddings J, Reichert H, Greene MT, et al. Evaluation of the association between Hospital Survey on Patient Safety Culture (HSOPS) measures and catheter-associated infections: results of two national collaboratives. BMJ Qual Saf 2016; Published Online First 24 May 2016.

12 Hessels AJ, Larson EL. Relationship between patient safety climate and standard precaution adherence: a systematic review of the literature. J Hosp Infect 2016;92:349-62.

13 Tad-y DB, Pierce RG, Pell JM, et al. Leveraging a redesigned morbidity and mortality conference that incorporates the clinical and educational missions of improving quality and patient safety. Acad Med 2016;91:1239-43.

14 National Patient Safety Foundation. Free from Harm: Accelerating Patient Safety Improvement Fifteen Years after To Err Is Human. http://c.ymcdn.com/sites/www.npsf.org/resource/ resmgr/PDF/Free_from_Harm.pdf. Published 19 May, 2016 (accessed 30 Aug 2016).

15 Bell SK, Delbanco T, Anderson-Shaw L, et al. Accountability for medical error: moving beyond blame to advocacy. Chest 2011;140:519-26.

16 Wachter RM. Personal accountability in healthcare: searching for the right balance. BMJ Qual Saf 2013;22:176-80.

17 Katz D, Detsky AS. Incorporating metacognition into morbidity and mortality rounds: The next frontier in quality improvement. J Hosp Med 2016;11:120-2.

18 Croskerry P. The importance of cognitive errors in diagnosis and strategies to minimize them. Acad Med 2003;78:775-80.

19 Redelmeier DA. Improving patient care. The cognitive psychology of missed diagnoses. Ann Intern Med 2005;142:115-20. 